\title{
Method for rapid identification of oral streptococci by PCR using 16S-23S ribosomal RNA intergenic spacer gene
}

\author{
Tomonori Hoshino*1, Tomohiro Izumi*2, Takashi Ooshima*2 and Taku Fujiwara*1 \\ *1 Division of Pediatric Dentistry, Department of Developmental and Reconstructive Medicine, \\ Nagasaki University Graduate School of Biomedical Sciences \\ 1-7-1 Sakamoto, Nagasaki 852-8588, JAPAN \\ *2 Department of Pediatric Dentistry, Osaka University Graduate School of Dentistry \\ 1-8 Yamada-oka, Suita, Osaka 565-0871, JAPAN
}

\begin{abstract}
Mutans group streptococci play an important role in the formation of dental caries, while mitis group streptococci are considered to be associated with subacute bacterial endocarditis. Both mitis and salivarius group streptococci, which are early colonizers in the human oral cavity, have an effect on the infection and colonization of mutans streptococci. In the present study, a rapid and exact PCR method to detect those oral streptococci was developed using the $16 \mathrm{~S}-23 \mathrm{~S}$ ribosomal RNA intergenic spacer gene, which is present between $16 \mathrm{~S}$ rRNA and 23S rRNA within the RNA operon. The PCR analysis was able to correctly identify mutans and mitis group streptococcal species. Our results indicate that the combined PCR analysis method established in the present study is useful for detecting oral streptococcal species, as well as in clinical applications to predict and prevent dental caries and etiological studies of streptococcal infective endocarditis.
\end{abstract}

Key words

16S-23S rRNA intergenic spacer gene,

Oral streptococci,

PCR,

Species identification

\section{Introduction}

Bacteria of the genus Streptococcus are subdivided into major phylogenic groups, which are the pyogenic, anginosus, bovis, salivarius, mitis, and mutans groups. Among them, some species of mutans group streptococci (Streptococcus mutans and Streptococcus sobrinus), mitis group streptococci (Streptococcus mitis, Streptococcus sanguinis, Streptococcus oralis, and Streptococcus gordonii), and salivarius group streptococci (Streptococcus salivarius, and others) are commonly found in the oral cavity. Further, mitis group streptococci are the most frequently isolated from the blood of patients with infective endocarditis ${ }^{1)}$, while mutans group streptococci have been implicated as the prime causative organisms of human dental caries ${ }^{2)}$. Since both mitis and salivarius group streptococci are

Received on March 31, 2005

Accepted on August 8, 2005 earlier colonizers in the human oral cavity ${ }^{3)}$, they likely have an effect on the infection and colonization of mutans streptococci. Although the cariogenic potential of mitis group streptococci is low, those species may play an antagonistic role in the development of dental caries ${ }^{4}$. The prevalence of mitis group streptococci has been shown to be different between caries-active and caries-inactive individuals ${ }^{5)}$, therefore, detection and identification of mutans and mitis group streptococci among the oral flora may be important for not only predicting and preventing dental caries but also the association with bacterial endocarditis.

The 16S-23S ribosomal RNA intergenic spacer (16S-23S spacer) gene is present between the $16 \mathrm{~S}$ and $23 \mathrm{~S}$ ribosomal RNA genes. This region is largely non-functional, thus it is likely to contain sequence variations from which taxonomically useful information might be obtained. Polymorphisms in the length and sequence of the $16 \mathrm{~S}-23 \mathrm{~S}$ spacer have been used for strain identification of Streptococcus 
Table 1 PCR Primers used in this study

\begin{tabular}{|c|c|c|c|}
\hline Target & Name & Primer sequence & Annealing temp. $\left({ }^{\circ} \mathrm{C}\right)$ \\
\hline Universal & TI-F & 5'GAAGTCGTAACAAGGTAGCCGT3' & 60 \\
\hline 16S-23S spacer & TI-R & 5'TGCCAGGGCATCCACC3' & \\
\hline S. mutans & MUT-F & 5'CTCСТTTCTAAGGAAAAACGCA3' & 60 \\
\hline 16S-23S spacer & MUT-R & 5'TGAACTCCAGACTGACTTATTAGAAAA3' & \\
\hline S. sobrinus & SOB-F & 5'CAATTACTTAGTAACGGAAGCACGTT3' & 60 \\
\hline 16S-23S spacer & SOB-R & 5'TTTGAGAATAGTTGAACTCCGTATCCT3' & \\
\hline S. sanguinis & SAN-F & 5'CACACGGAATGCACTTGAGTC3' & 65 \\
\hline 16S-23S spacer & SAN-R & 5'CTAGTCAAACTAGATAATGGAGCCT3' & \\
\hline S.oralis and S.mitis & $\mathrm{O} / \mathrm{M}-\mathrm{F}$ & 5'AGGATAAGGAACTGCACATTGGTC3' & 68 \\
\hline $16 \mathrm{~S}-23 \mathrm{~S}$ spacer & $\mathrm{O} / \mathrm{M}-\mathrm{R}$ & 5'TGCATTACTTGGTGATCTCTCACC3' & \\
\hline S. gordonii & GOR-F & 5'AAACGGAATGCACGATGGAGTCTTG3' & 64 \\
\hline $16 \mathrm{~S}-23 \mathrm{~S}$ spacer & GOR-R & 5'CTCATTAATATTCACAGAGTTTCGG3' & \\
\hline
\end{tabular}

anginosus and Streptococcus constellatus belonging to "milleri group" streptococci ${ }^{6}$. However, few sequence data for the 16S-23S spacer from mutans and mitis group streptococci in samples from human oral cavities are available.

The purpose of this study was to analyze the $16 \mathrm{~S}-23 \mathrm{~S}$ spacer genes from oral streptococci, in order to design species specific polymerase chain reaction (PCR) primers based on the sequence data, and to develop useful methods to detect and identify oral streptococcal species in the human oral cavity.

\section{Materials and Methods}

\section{Bacterial strains}

The bacterial strains used in this study were S. mutans MT8148 (serotype c), GS5 (c), MT4245 $(e), \operatorname{MT} 4467(e), \operatorname{MT} 4251(f)$, and OMZ175 $(f)$, S. sobrinus OMZ176 $(d), \mathrm{B} 13(d), 6715(g)$, and MT10186 (g), S. salivarius NCTC 8618, and HHT, S. sanguinis ATCC 10556, and ST3, S. oralis ATCC 10557, and SK23, S. gordonii ATCC 10558, and SK51, and S.mitis SK24, and ATCC 903, which came from our culture collection. Organisms were routinely cultured in brain heart infusion broth (Difco Laboratories, Detroit, Mich., USA).

\section{Sequence analysis of the 16S-23S rRNA intergenic spacer genes from oral streptococci}

For sequencing of the 16S-23S spacer genes, primers TI-F and TI-R (Table 1) were designed from the conserved region of $16 \mathrm{~S} \mathrm{rRNA}^{7)}$ and $23 \mathrm{~S}_{\mathrm{rRNA}^{6}}$.
Preparation of chromosomal DNA from oral streptococci was performed as described previously ${ }^{8}$. PCR amplification was performed using chromosomal DNA from each oral streptococcal strain as a template. The PCR product was prepared using a Centricon 100 spin column (Millipore, Bedford, Mass., USA) for a DNA-sequencing template. A dideoxy dye termination reaction was performed using an ABI PRISM cycle sequencing kit (Applied Biosystems, Foster City, Calif., USA) in a GeneAmp 2400 thermal cycler (Applied Biosystems), following manufacturer's protocols. The product was then analyzed using an automated DNA sequencer model 373 (Applied Biosystems).

\section{Sequence analysis and design of primers}

Analyses of sequence data and the multiple-sequence alignment were performed with nucleic acid sequence and protein sequence analysis software, GeneWorks (IntelliGenetics Inc., Mountain View, Calif., USA). The primers were designed with the help of PrimerExpress software (Applied Biosystems).

\section{PCR condition and agarose gel electrophoresis}

PCR assays of the 16S-23S spacer genes were carried out in $20 \mu \mathrm{l}$ of reaction mixture containing 0.5 U AmpliTaq Gold DNA polymerase (Applied Biosystems), $0.5 \mu \mathrm{M}$ of oligonucleotide primers, $10 \mathrm{ng}$ of oral streptococcal chromosomal DNA, and $1.5 \mathrm{mM} \mathrm{MgCl}$, following the manufacturers' protocols. Amplification was performed with the GeneAmp 2400 thermal cycler using the following 
16S Ribosomal RNA >

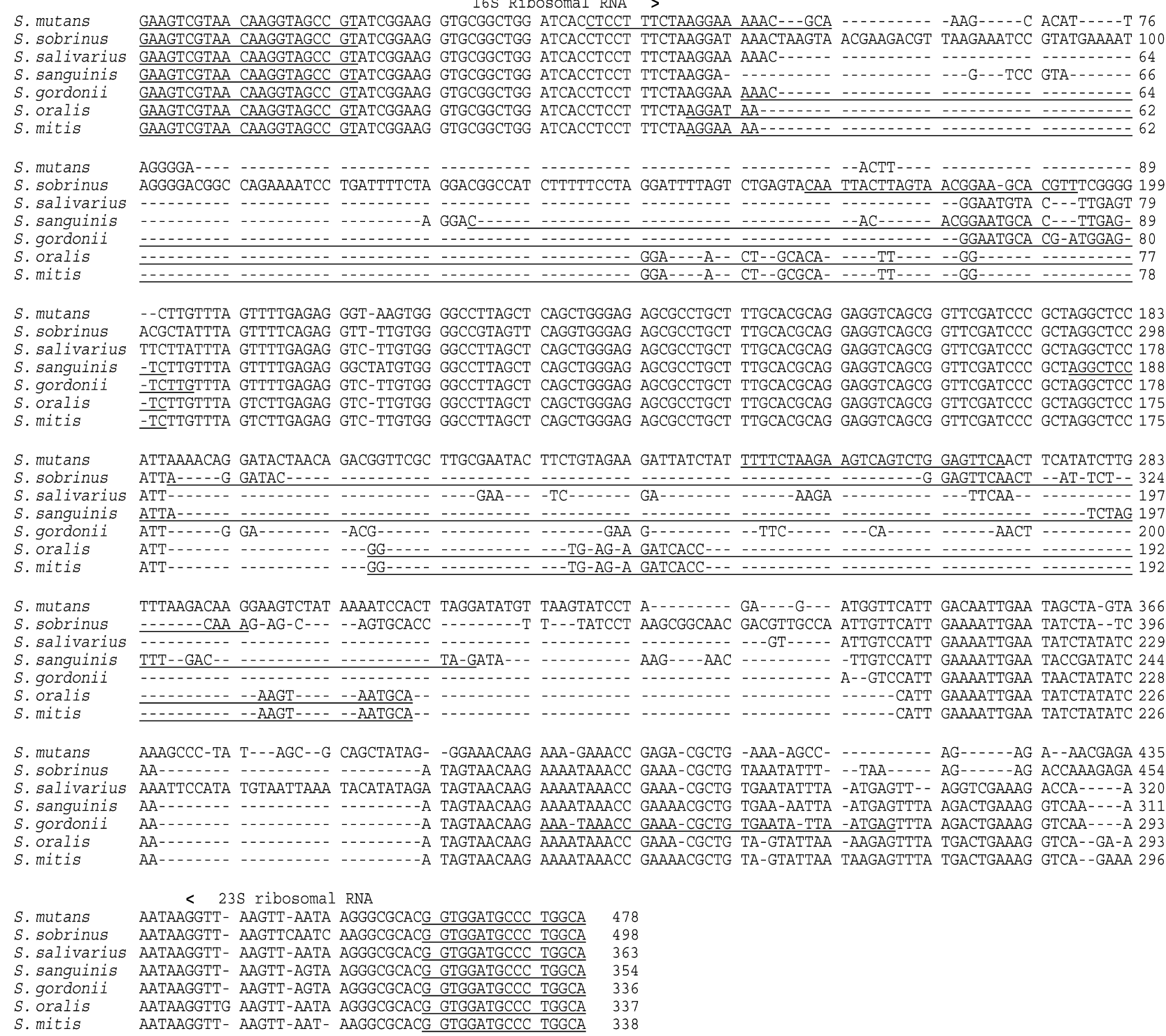

Fig. 1 Multiple alignment of 16S-23S ribosomal RNA intergenic spacer sequence of oral streptococci

As no inter-strain differences were observed among the tested strains, only 1 sequence is shown. The nucleotide sequences have been deposited in the DDBJ as follows: S. mutans strains MT8148, AB050989; MT4245, AB050988; MT4251, AB050990; S. sobrinus strains 6715, AB051014; OMZ176, AB051015; S. salivarius NCTC 8618, AB051016; S. sanguinis ATCC 10556, AB051017; S. oralis ATCC 10557, AB051018; S.gordonii ATCC 10558, AB051019; and S.mitis SK24, AB051020. Gaps are indicated by hyphens and the sequences of the designed PCR primers are underlined. End point of $16 \mathrm{~S}$ ribosomal RNA and start point of 23S ribosomal RNA sequence was indicated by " $>$ " and " $<$ ", respectively.

parameters; a preincubation step at $95^{\circ} \mathrm{C}$ for 9 minutes, followed by 30 cycles of a denaturing step at $94^{\circ} \mathrm{C}$ for 30 seconds, and a primer-annealing and extension step at $60^{\circ} \mathrm{C}$ to $68^{\circ} \mathrm{C}$ for 1 minute. The primer-annealing and extension temperature was changed with every primer set (Table 1). The PCR products were visualized by $2.0 \%$ agarose gel electrophoresis using ethidium bromide staining. For molecular weight standards, a $100 \mathrm{bp}$ DNA ladder (Promega, Madison, Wis., USA) was used.

\section{Nucleotide sequence accession numbers}

The nucleotide sequences of the 16S-23S rRNA intergenic spacer gene have been deposited in the DDBJ database as follows: S. mutans strains MT8148, AB050989; MT4245, AB050988; MT4251, AB050990; S. sobrinus stains 6715, AB051014; OMZ176, AB051015; S. salivarius NCTC 8618, 


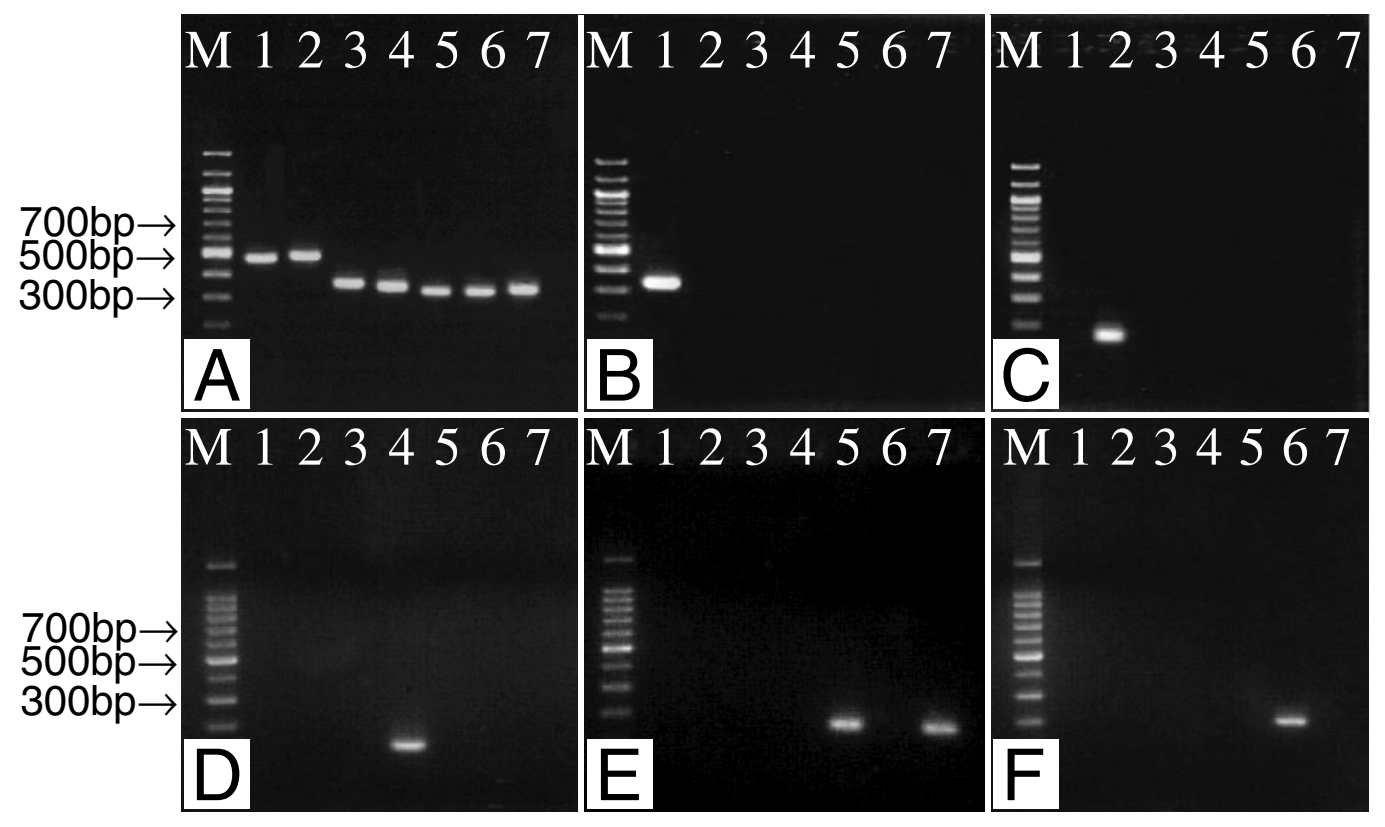

Fig. 2 PCR amplification of 16S-23S ribosomal RNA intergenic spacer genes

PCR amplification was performed with the primer sets from whole 16S-23S ribosomal RNA genes (A), as well as species-specific primer sets against S. mutans (B), S. sobrinus (C), S. sanguinis (D), S. oralis and S. mitis (E), and S. gordonii (F). Lanes: M, 100bp molecular weight marker; 1, S. mutans; 2, S. sobrinus; 3, S. salivarius; 4, S. sanguinis; 5, S. oralis; 6, S. gordonii; and 7, S.mitis.

AB051016; S. sanguinis ATCC 10556, AB051017; S. oralis ATCC 10557, AB051018; S. gordonii ATCC 10558, AB051019; and S. mitis SK24, AB051020.

\section{Results}

\section{DNA sequence analyses of the $16 \mathrm{~S}-23 \mathrm{~S}$ spacer genes}

The DNA sequences of the 16S-23S spacer genes from S. mutans, S. sobrinus, S. salivarius, S. sanguinis, S. oralis, S. gordonii, and S. mitis found to be $388 \mathrm{bp}$, $407 \mathrm{bp}, 273 \mathrm{bp}, 264 \mathrm{bp}, 246 \mathrm{bp}, 246 \mathrm{bp}$, and $249 \mathrm{bp}$, respectively. There was no significant difference among serotype $c / e / f$ of $S$. mutans and $d / g$ of S. sobrinus strains, and no inter-strain difference was observed in the tested oral streptococci (data not shown). The multiple alignment revealed that the 16S-23S spacer genes were different among the species except between $S$. oralis and S. mitis, which were shown to be nearly identical (Fig. 1).

\section{Design of specific primers using the 16S-23S spacer genes}

The aligned sequences of the $16 \mathrm{~S}-23 \mathrm{~S}$ spacer genes were manually compared, and species-specific primers based on the alignment of the $16 \mathrm{~S}-23 \mathrm{~S}$ spacer genes were designed and synthesized, as shown in the Table 1 and Fig. 1. However, the species-specific region of the 16S-23S spacer gene from S. salivarius was too short to design a species-specific PCR primer set.

\section{PCR amplification of the 16S-23S spacer genes}

Figure 2 shows representative results of PCR amplification of the 16S-23S spacer genes. Using primers based on the 16S-23S spacer gene, speciesspecific amplification was observed for all of the species, except for S. salivarius. PCR products were amplified from both S.oralis and S.mitis chromosomal DNA with the primer set of $\mathrm{O} / \mathrm{M}-\mathrm{F}$ and $\mathrm{O} / \mathrm{M}-\mathrm{R}$, which was against the identical 16S$23 \mathrm{~S}$ spacer genes of S. oralis and S. mitis. Each species-specific amplification was confirmed by direct sequencing of the amplicon. The concentration of streptococcal DNA available for the PCR analysis was approximately $50 \mathrm{pg} / \mathrm{ml}$.

\section{Discussion}

In the present study, we designed primers corresponding to the $16 \mathrm{~S}-23 \mathrm{~S}$ spacer genes to enable identification of oral streptococci using a simple 
and reliable PCR method. Species identification of streptococci is usually determined from phenotypic characteristics such as colony morphology, hemolysis, carbohydrate fermentation, and serological properties ${ }^{9}$. Since the biochemical, physiological, serological characteristics of mitis group streptococci are very similar ${ }^{9}$, classification of those strains has been difficult. In addition, many nucleic acid-based typing methods have been applied to studies of the epidemiology ${ }^{10,11)}$, including restriction endonuclease analysis ${ }^{12)}$, probe-based fingerprinting ${ }^{13,14)}$, and PCRbased fingerprinting ${ }^{15,16}$. PCR methods offer several advantages over other nucleic acid-based typing methods, such as: speed, the need for fewer cells, and protocols that involve fewer steps.

Although DNA sequencing is the best method of quantitatively determining bacterial taxonomy, it requires an expensive automated DNA sequencer apparatus and complex experimental procedures. A comparison of 16S rRNA sequences is well established as a standard method for the identification of bacterial species, genera, and families, though it may not be useful for subtyping. Since the homology of 16S rRNA sequences among oral streptococcal species is more than $96 \%$, it is impossible to design a species-specific PCR primer for that region.

The 16S-23S spacer gene is a stretch of DNA that lies between the $16 \mathrm{~S}$ and $23 \mathrm{~S}$ rRNA region, and is under minimal selective pressure because of its non-coding function, and should have accumulated a higher percentage of mutations than rRNA genes ${ }^{17}$. Therefore, it may be useful for accurate identification of species, due to the enhanced variations between species within a genus.

The multiple sequence alignment of the 16S-23S spacer genes revealed that this region was a speciesspecific region in the present oral streptococci, except for S.oralis and S.mitis. Using a PCR analysis method with the primers for the 16S-23S spacer genes, we were able to identify all of the present oral streptococcal species, except for S. oralis and S. mitis. In addition, no species-specific primer against $S$. salivarius was found.

Recently, we developed a PCR method that utilizes the hypervariable region of the gene encoding glucosyltransferase $(g t f)$ as the target gene ${ }^{18}$. Nearly all oral streptococci, with the exception of S.mitis, produce extracellular polysaccharide ${ }^{9)}$ and possess the $g t f$ gene $^{19)}$. Therefore, the differentiation of S. mitis and S. oralis as well as the detection of S. salivarius can be performed by a combination of these gtf based primers. In the epidemiologic study of sepsis and endocarditis caused by oral streptococci, the identification of mitis group streptococci is very important, since it is reported that viridans streptococci, including mitis group streptococci, is most frequently associated with infective endocarditis ${ }^{20)}$. Thus, it is thought that the method presented here is very available for the clinical research of endocarditis, since it is possible to distinguish those streptococci in species level.

Several investigators have developed PCR methods to detect the species of mutans group streptococci using the dextranase gene ${ }^{21}$, and the insoluble glucan synthesizing $g t f$ gene $^{22)}$. However, the information presented in those studies is only useful for identifying species of mutans group streptococci.

We reported that glucosyltransferase from S. oralis, one of early colonizing streptococci, plays an important role in the inhabitation of $S$. mutans on tooth surface ${ }^{8)}$. This result indicated that commensal streptococci were necessary to the formation of cariogenic dental plaque. Thus, it is important to perform research of symbiosis among mutans group streptococci and the other group streptococci. Especially, the understanding of the change of oral microflora from predentate infant to dentate child might be useful for the control of the inhabitation of mutans streptococci and the prevention of caries in early childhood. It seems to be very significant that the combined method, described above, is applied in clinical investigations of pediatric dentistry.

The development of real-time PCR methods has made it possible to directly determine the amount of bacteria in a sample without culturing, such as Porphyromonas gingivalis ${ }^{23}$. In addition, using a real-time reverse-transcription/polymerase reaction, we previously determined the expression of 3 different $g t f$ genes $^{24)}$. Currently, we are attempting quantitative analyses of streptococci in saliva and plaque, in order to determine which streptococcal species affect to the infection and colonization of mutans group streptococci. We were able to identify oral streptococci with the PCR analysis developed in the present report, though further studies are required to establish a quantitative method of streptococcal species identification using a real-time PCR method.

\section{Acknowledgments}

This work was supported in part by a grant-in aid 
from the ministry of Education, Science and Sports of Japan (15390639, 16592050 and 16659574).

\section{References}

1) Douglas, C.W.I., Heath, J., Hampton, K.K. and Preston, F.E.: Identity of viridans streptococci isolated from cases of infective endocarditis. $J$ Med Microbiol 39: 179-182, 1993.

2) Hamada, S. and Slade, H.D.: Biology, immunology, and cariogenicity of Streptococcus mutans. Microbiol Rev 44: 331-384, 1980.

3) Pearce, C., Bowden, G.H., Evans, M., Fitzsimmons, S.P., Johnson, J., Sheridan, M.J., Wientzen, R. and Cole, M.F.: Identification of pioneer viridans streptococci in the oral cavity of human neonates. J Med Microbiol 42: 67-72, 1995.

4) Loesche, W.L.: Role of Streptococcus mutans in human dental decay. Microbiol Rev 50: 353-380, 1986.

5) Nyvad, B. and Kilian, M.: Comparison of the initial streptococcal microflora on dental enamel in cariesactive and in caries-inactive individuals. Caries Res 24: 267-272, 1990.

6) Whiley, R.A., Duke, B., Hardie, J.M. and Hall, L.M.C.: Heterogeneity among 16S-23S rRNA intergenic spacers of species within the 'Streptococcus milleri group'. Microbiology 141: 1461-1467, 1995.

7) Bentley, R.W., Leigh, J.A. and Collins, M.D.: Intrageneric structure of Streptococcus based on comparative analysis of small-subunit rRNA sequences. Int J Syst Bacteriol 41: 487-494, 1991.

8) Fujiwara, T., Hoshino, T., Ooshima, T., Sobue, S. and Hamada, S.: Purification, characterization, and molecular analysis of the gene encoding glucosyltransferase from Streptococcus oralis. Infect Immun 68: 2475-2483, 2000.

9) Kilian, M., Mikkelsen, L. and Henrichsen, J.: Taxonomic study of viridans streptococci: Description of Streptococcus gordonii sp. nov. and emended descriptions of Streptococcus sanguis (White and Niven 1946), Streptococcus oralis (Bridge and Sneath 1982), and Streptococcus mitis (Andrews and Horder 1906). Int J Syst Bacteriol 39: 471-484, 1989.

10) Caufield, P.W. and Walker, T.M.: Genetic diversity within Streptococcus mutans evident from chromosomal DNA restriction fragment polymorphisms. J Clin Microbiol 27: 274-278, 1989.

11) Swaminathan, B. and Matar, G.M.: Molecular typing methods. In: Diagnostic Molecular Microbiology: Principles and Applications. (Persing, D.H., Smith, T.F., Tenover, F.C. and White, T.J. eds.) American Society for Microbiology, Washington, D.C., 1993, pp. 26-50.

12) Kulkarni, G.V., Chan, K.H. and Sandham, H.J.: An investigation into the use of restriction endonuclease analysis for the study of transmission of mutans streptococci. J Dent Res 68: 1155-1161, 1989.

13) Cangelosi, G.A., Iverson, J.M., Zuo, Y., Oswald, T.K. and Lamont, R.J.: Oligonucleotide probes for mutans streptococci. Mol Cell Probes 8: 73-80, 1994.

14) Saarela, M., Alaluusua, S., Takei, T. and Asikainen, S.: Genetic diversity within isolates of mutans streptococci recognized by an rRNA gene probe. J Clin Microbiol 31: 584-587, 1993.

15) Li, Y. and Caufield, P.W.: Arbitrarily primed polymerase chain reaction fingerprinting for the genotypic identification of mutans streptococci from humans. Oral Microbiol Immunol 13: 17-22, 1998.

16) Shiroza, T., Shinozaki, N., Watanabe, T., Ikemi, T., Fukushima, K. and Abiko, Y.: Rapid isolation of chromosomal DNA from oral streptococci and polymerase chain reaction-oriented restriction fragment-length polymorphism analysis for genetic heterogeneity. Oral Microbiol Immunol 13: 11-16, 1998.

17) Tyrrell, G.J., Bethune, R.N., Willey, B. and Low, D.E.: Species identification of enterococci via intergenic ribosomal PCR. J Clin Microbiol 35: 1054-1060, 1997.

18) Hoshino, T., Kawaguchi, M., Shimizu, N., Hoshino, N., Ooshima, T. and Fujiwara, T.: PCR detection and identification of oral streptococci in saliva samples using $g t f$ genes. Diagn Microbiol Infect Dis 48: 195-199, 2004.

19) Monchois, V., Willemot, R.M. and Monsan, P.: Glucansucrases: mechanism of action and structurefunction relationships. FEMS Microbiol Rev 23: 131-151, 1999.

20) Tomas Carmona, I., Diz Dios, P., Limeres Posse, J., Gonzalez Quintela, A., Martinez Vazquez, C. and Castro Iglesias, A.: An update on infective endocarditis of dental origin. J Dent 30: 37-40, 2002.

21) Igarashi, T., Yamamoto, A. and Goto, N.: Direct detection of Streptococcus mutans in human dental plaque by polymerase chain reaction. Oral Microbiol Immunol 5: 294-298, 1996.

22) Oho, T., Yamashita, Y., Shimazaki, Y., Kushiyama, M. and Koga, T.: Simple and rapid detection of Streptococcus mutans and Streptococcus sobrinus in human saliva by polymerase chain reaction. Oral Microbiol Immunol 15: 258-262, 2000.

23) Lyons, S.R., Griffen, A.L. and Leys, E.J.: Quantitative real-time PCR for Porphyromonas gingivalis and total bacteria. J Clin Microbiol 38: 2362-2365, 2000.

24) Fujiwara, T., Hoshino, T., Ooshima, T. and Hamada, S.: Differential and quantitative analyses of mRNA expression of glucosyltransferases from Streptococcus mutans MT8148. J Dent Res 81: 109-113, 2002. 